\title{
Comparison of two ELISA-based methods for the detection of microcystins in blood serum
}

\author{
Alexandra H. Heussner ${ }^{a}$, Isabell Winter ${ }^{a}$, Stefan Altaner ${ }^{\mathrm{a}}$, Lisa Kamp ${ }^{\mathrm{b}}$, Fernando Rubio ${ }^{\mathrm{b}}$, \\ Daniel R. Dietrich ${ }^{\mathrm{a}, *}$ \\ ${ }^{a}$ Human and Environmental Toxicology, University of Konstanz, Universitätsstrasse 10, 78457 Konstanz, Germany \\ ${ }^{\mathrm{b}}$ Abraxis LLC, 54 Steamwhistle Drive, Warminster, PA 18974, USA
}

Keywords:

ELISA

Microcystin

Human serum

Sensitivity

Recovery

\section{A B S T R A C T}

Microcystins (MCs) are cyanobacterial toxins which place the public at risk via exposure to MC contam inated water, food or algal food supplements. Subsequent to the fatal intravenous exposure of dialysis patients in Caruaru, Brazil, several techniques (LC MS, GC MS and ELISA) were adapted to detect MCs in human serum. As patients chronically exposed to low concentrations of MCs also present with very low MC serum levels, only LC MS methodology would appear to allow detection of these MC levels. How ever, LC MS detection depends on the availability of respective MC congener standards and the levels of non covalently bound MC in the sample. In contrast, immunological techniques, e.g. MC ELISA poten tially could detect even covalently bound MC, provided the MC antibody was raised against an epitope found in nearly all of the MC congeners. As the Adda side chain moiety is present in nearly all of the MC congeners known to date, the anti Adda antibodies, when applied in Adda ELISAs, could represent a relatively simple and robust technique for the qualitative and quantitative determination of $\mathrm{MC}$ in human serum.

The aim of the current study was to determine whether commercially available Adda ELISAs and their respective sample preparation methods would allow MC quantification in human serum. The Adda ELISA (polyclonal antibody) and the Adda ELISA (monoclonal antibody) kit for serum (Serum ELISA) were used for determination of the concentration dependent recovery of MCs in MC spiked serum.

Human serum samples were spiked with varying concentrations of MCs (MC LR, YR, RR, LA, LW, LF and defined MC mixtures) and extracted using two different methods. MC spiked bovine serum and stan dard cell culture medium containing $10 \%$ FBS served to investigate potential matrix effects. Inter labora tory comparison was performed allowing identification of potential sources of error.

The results suggest that both ELISAs are suitable tools for the analysis of MCs in human blood serum although both also displayed some weaknesses notably the time needed for sample preparation or the overestimation of some specific MC congener concentrations. Based on the ELISA detection ranges, sam ple concentration and/or MC spiking may be required for detection of low levels of MCs in human blood.
Abbreviations: $\mathrm{MeOH}$, methanol; SPE, solid phase extraction; MC, microcystin; LOD, limit of detection; FBS, fetal bovine serum; RT, room temperature; DMEM, Dulbecco's modified Eagle medium; PPIA, protein phosphatase inhibition assay; HPLC, high performance liquid chromatography; LC-MS, liquid chromatographymass spectrometry; ELISA, enzyme-linked immunosorbent assay; PP, polypropylene; LDPE, low-density polyethylene; HDPE, high-density polyethylene.

* Corresponding author. Tel.: +497531 883518.

E-mail addresses: alexandra.heussner@uni-konstanz.de (A.H. Heussner), isabell. winter@uni-konstanz.de (I. Winter),stefan.altaner@uni-konstanz.de (S. Altaner), lkamp@abraxiskits.com (L. Kamp), frubio@abraxiskits.com (F. Rubio), daniel. dietrich@uni-konstanz.de (D.R. Dietrich).

\section{Introduction}

Microcystins (MCs) are toxic cyclic hepta peptides produced by certain cyanobacteria, predominantly by the cyanobacterial genera Anabaena, Microcystis, Nostoc and Planktothrix [1]. Toxic algae blooms are a problem worldwide found in all types of water bodies, they appear to increase in frequency with climate change associated higher average annual ambient temperatures. Although many countries in the Western world introduced monitoring strategies, developing countries especially face increasingly 
harmful algal blooms due to intensified agriculture and industry, which pose a significant threat to public health [2].

More than 80 MC congeners have been identified to date [3], whereby MC LR is the best characterized congener and is also clas sified as a 2B carcinogen by the International Agency for Research on Cancer (IARC) [4]. As cyanobacteria are able to produce different MC congeners simultaneously, affected waters are always contami nated with a whole range of different MCs [5]. This was shown for example for the cyanobacterium Microcystis aeruginosa PCC 7820, where MC LR, LY, LW and LF were detected [6]. Concomitant with the structural differences the toxicity of the congeners vary, whereby a priori the toxicity appears to be governed more by kinetic rather than dynamic differences amongst congeners $[7,8]$. While the kinetics of MC uptake appears primarily dependent on trans mem brane transport by organic anion transporting peptides (OATPs) [9], the toxicodynamics are characterized by a highly specific inhibition of ser/thr protein phosphatases (PPs) [10 12]. Corresponding with a first pass effect and a high expression of OATPs and PPs, the imme diate result of acute exposure to MCs of humans and other mamma lians is liver damage followed by a hepatic renal syndrome and multi organ failure, as exemplified by dialysis patients in Caruaru, Brazil, in 1996 [13]. The latter patients were exposed to MCs in dial ysis centers with dialysis water contaminated with MCs.

Following the severe morbidity and ensuing mortality of patients, sera from these patients were analyzed repeatedly for the presence of MCs by various investigators [14 17]. The analyses included several detection methods such as ELISA, LC MS and GC MS as well as diverse sample preparation methods. While one group of investigators found MC serum concentrations ranging from $\leqslant 0.16$ to $0.96 \mu \mathrm{g} / \mathrm{L}$ [17], others detected a mean serum concentration of $2.2 \mu \mathrm{g} / \mathrm{L}$ [18]. Single values were as high as $133 \mu \mathrm{g} / \mathrm{L}$ [15]. The pre dominant congeners in the patient serum detected were MC LR, YR, and AR [19]. To date, most of the available data on the detection of MCs in human serum is associated with the Caruaru event.

Additionally, a second, less severe MC exposure during hemod ialysis occurred in Rio de Janeiro, Brazil, in 2001. From this event, affected patients were screened amongst other things for MC in serum [20]. Thirteen out of 44 patients showed detectable MC lev els with a maximum median level of $0.61 \mu \mathrm{g} / \mathrm{L}$ [20].

Beyond the accidental intravenous exposure with the associated acute effects, there are more common routes of human exposure to MCs. These include the "voluntary" uptake of MC contaminated algal food supplements, consumption of contaminated food (e.g. fish and crayfish from water bodies with known frequent occur rences of algal blooms or vegetables and salads watered with con taminated water) [21,22], as well as MC exposure via contaminated drinking water, thereby leading to chronic MC exposure. Indeed, MCs and other toxins are frequently detected in algal food supple ments, ranging from approximately $0.15 .7 \mu \mathrm{g} \mathrm{MC} \mathrm{LR}_{\text {eq }}$ per gram dry weight $[22,23]$.

To date, only very limited data are available on human contami nation levels following exposure to contaminated drinking water, food or food supplements. For example, Chen and co workers inves tigated fishermen at Lake Chaohu, China, chronically exposed to MCs via drinking water, aerosols and consumption of aquatic food. MCs were detected in serum samples at an average of $0.39 \mu \mathrm{g} \mathrm{MC} \mathrm{LR}$ eq $/ \mathrm{L}$ [24]. Similarly, Li and co workers analyzed samples from children exposed to contaminated drinking water and aquatic food and found serum concentrations ranging from 0.4 to $1.3 \mu \mathrm{g} \mathrm{MC} \mathrm{LR}_{\mathrm{eq}} / \mathrm{L}$ [25].

In light of the 2B human carcinogen classification of MC LR by the IARC [4] and in view of the recent suggestion that MC mediated neurotoxicity could predispose for human neurodegenerative dis eases $[7,8]$, any exposure with MCs must be regarded as potentially harmful to humans. Thus the routine robust and sensitive detec tion of low levels of microcystins in human blood serum will be the key to identifying patients potentially at high risk for diseases associated with chronic MC exposure. ELISA based methods have been proven to be suitable tools for the detection of MCs in water, in cyanobacterial cells and in exposed human, animal and plant materials, although some methodological constraints and require ments are essential for the outcome [26]. Consequently, this study aimed to evaluate two different commercially available ELISAs, with their respective sample preparation methods, with respect to their applicability for the reliable detection of MC LR, YR, RR, LA, LW, LF as well as defined MC mixtures in human blood. In addition, the suitability of these methods for the detection of MCs in in vitro applications was tested using bovine serum (FBS), which is a typical ingredient in cell culture media, and a complete standard cell culture medium containing 10\% FBS.

\section{Materials and methods}

\subsection{Materials}

Unless otherwise stated, materials were purchased as follows: Abraxis, Warminster, PA, USA (ELISA kits), PAA Laboratories GmbH, Cölbe, Germany (FBS gold, DMEM), Waters GmbH, Eschborn, Ger many (Oasis ${ }^{\circledR}$ HLB 6 cc (200 mg) extraction cartridges), Enzo Life Sciences GmbH, Lörrach, Germany (MC LR, MC YR, MC RR, MC LA, MC LW and MC LF), and Sigma Aldrich GmbH, Seelze, Ger many (all other chemicals).

\subsection{Preparation of samples}

MC LR and MC RR were dissolved in $100 \% \mathrm{MeOH}$ to a nominal concentration of $200 \mu \mathrm{M}$ and the real concentration was determined via detection of absorbance at $\lambda_{238} \mathrm{~nm}$ and using the molar absorp tion coefficient of $39,800 \mathrm{~mol} \mathrm{~L}^{-1} \mathrm{~cm}^{-1}[27,28]$ after calibration of the spectrophotometer with potassium dichromate in $1 \mathrm{mM}$ per chloric acid [29]. For lack of other validated coefficients, the other MC congeners were prepared the same way. Although some investi gators used specific extinction coefficients (Wayne Carmichael, per sonal communication), using the same coefficient for the major MC congeners represents a widely accepted approach because the dif ferences seem to be rather small (personal communication with Jus si Meriluoto and Linda Lawton). Three different matrices were chosen as samples, i.e. human serum, FBS and standard cell culture medium containing $10 \%$ FBS. These samples were mixed with the respective $\mathrm{MC}$ congener(s) to yield a range of final concentrations (between 1 and $200 \mu \mathrm{g} / \mathrm{L}$, see Results section for details) and stored at $-20^{\circ} \mathrm{C}$ until use. All samples were prepared at least in triplicate.

\subsection{Sample extraction for the detection in the Adda ELISA}

The extraction procedure is summarized in Fig. 1.

\subsection{Purification of toxins from extracts via SPE for the detection via the} Adda ELISA

Solid phase extraction (SPE) was performed using Oasis HLB 6 cc (200 mg) cartridges, according to the manufacturer's instructions (Waters $\mathrm{GmbH}$, Eschborn, Germany), in order to purify and concen trate toxins in the extracts (Fig. 1). Briefly, SPE columns were precon ditioned with $100 \% \mathrm{MeOH}(2 \mathrm{ml})$ and equilibrated with deionized water $(2 \mathrm{ml})$. Extracts were applied to the columns and the columns then washed with $30 \%(\mathrm{v} / \mathrm{v}) \mathrm{MeOH}$ in $0.1 \mathrm{M}$ acetic acid $(3 \times 2 \mathrm{ml})$. $80 \% \mathrm{MeOH}(5 \mathrm{ml})$ was employed for MC elution from the column. Collected eluates from the same sample were combined and evapo rated to dryness under a stream of nitrogen. Dried samples were resuspended in $500 \mu \mathrm{l}$ \% (v/v) aqueous $\mathrm{MeOH}$, vigorously mixed and sonicated for $5 \mathrm{~min}$. The final samples were stored at $-20^{\circ} \mathrm{C}$ until further use. 


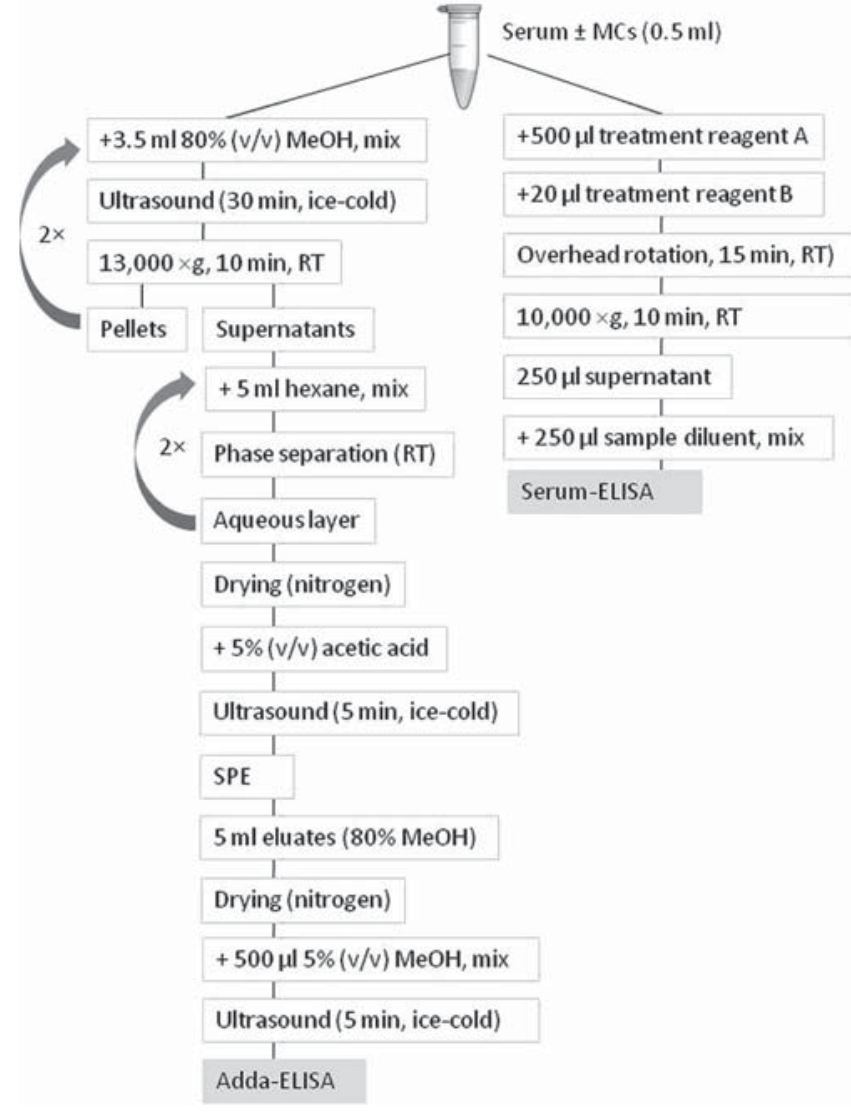

Fig. 1. Overview of sample preparation from spiked human serum.

\subsection{Adda ELISA}

Extracts, prepared via methanol extraction and SPE, were ana lyzed using a commercially available Adda ELISA kit ("Microcys tins/Nodularins (ADDA) ELISA", Abraxis LLC, Warminster, PA, USA; cat\# 520011) according to manufacturer's instructions. This indirect competitive ELISA recognizes specifically the Adda moiety present in all known MC congeners via a polyclonal antibody [30], thus detecting MCs independent of the congener. All samples were ana lyzed in triplicate on each plate and three independent samples were generated for all analyses. Before use in ELISA samples were diluted to fit the linear range of the assay $(0.155 \mu \mathrm{g} / \mathrm{L})$, with all sam ples above $1 \mu \mathrm{g} / \mathrm{L}$ being diluted to nominally $1 \mu \mathrm{g} / \mathrm{L}$. The resulting MC concentrations were calculated from the MC LR standard cali bration curve and are presented as MC LR equivalents (MC $\left.L_{\mathrm{eq}}\right)$. Samples, which were out of the range of the standard curve of each ELISA, were considered to be below the limit of detection $(<\mathrm{LOD})$. The test sensitivity and reproducibility was given by the manufac turer with limit of detection (LOD) of $0.1 \mu \mathrm{g} \mathrm{MC} \mathrm{LR/L} \mathrm{and} \mathrm{coeffi}$ cients of variation (CVs) of $<10 \%$ and $<15 \%$ for standards and samples, respectively. No information was available with regard to the recovery of MCs, however a theoretical range of recovery of $80120 \%$ was set based on own preliminary data and detected CVs and other observations from the manufacturer and other investiga tors $[30,31]$.

\subsection{Sample preparation for the detection of MCs with the Serum ELISA kit}

Serum samples were extracted and analyzed using a commer cially available Microcystin ELISA kit for serum (Abraxis LLC, War minster, PA, USA; cat\# 522031) according to manufacturer's instructions (Fig. 1). This direct competitive ELISA recognizes specifically the Adda moiety present in all known MC congeners via a monoclonal antibody [32], thus detecting MCs independent of the congener. All samples were diluted to nominally $0.5 \mu \mathrm{g} / \mathrm{L}$ to fit into the standard calibration range of this direct competitive Serum ELISA (0.1 $1.0 \mu \mathrm{g} \mathrm{MC} \mathrm{LR/L),} \mathrm{the} \mathrm{resulting} \mathrm{MC} \mathrm{concentra}$ tions were calculated from the MC LR standard calibration curve and are presented as $M C L R_{\text {eq. }}$. Samples, which were out of the range of the standard curve of each ELISA, were considered to be below the limit of detection $(<\mathrm{LOD})$. The test sensitivity and reproducibil ity was given by the manufacturer with an LOD of $0.40 \mu \mathrm{g} \mathrm{MC} \mathrm{LR/L}$ and CVs of $10 \%$ and $<15 \%$ for standards and samples, respectively. The sample recovery from human serum was indicated as being 70 130\%.

\subsection{Samples for the comparison of different storage vessels}

MC LR, LW or LF were spiked into ELISA diluent at $1 \mu \mathrm{g} / \mathrm{L}$ in glass vials and were then transferred into different vessels: polypro pylene microcentrifuge tubes (PP (E), very slightly opaque, Eppen dorf, cat\# 022363352), polypropylene microcentrifuge tubes (PP (T), clear, Thomas Scientific, cat\# 1232G82), polypropylene micro centrifuge tubes (PP (V), clear, VWR, cat\# 20170 355), low density polyethylene (LDPE) bottles (Wheaton, cat\# W242831 A), high density polyethylene (HDPE) bottles (Nalgene, cat\# 0331325A), and glass vials with Teflon lined caps (La Pha Pack, cat\# 13090222). These samples were stored at $4{ }^{\circ} \mathrm{C}$ and tested after $24 \mathrm{~h}$ and $48 \mathrm{~h}$ using the monoclonal based ELISA as described above. For each vessel type, one aliquot was analyzed with no mixing whereas the other aliquot was analyzed with mixing prior to pipet ting onto the microtiter plate.

\subsection{Samples for inter laboratory comparison}

Three types of spiked samples were analyzed in each of the two laboratories: (1) samples using the lab's own serum lot and the own MC stock solutions, (2) samples using the lab's own serum lot and the MC stock solutions of the other lab and (3) samples completely prepared by the other lab. These samples were pro duced using MC LR, LW and LF and $10 \mu \mathrm{g} / \mathrm{L}$ and $100 \mu \mathrm{g} / \mathrm{L}$.

\subsection{Calculations and statistical data analysis}

ELISA data were processed according to manufacturer's instruc tions. Briefly, absorbance values were calculated as percentage of negative control for each plate and compared with the calibration data achieved via linear regression analysis. If appropriate, means \pm SDs were calculated from at least three independent sam ples conducted in triplicate. These recovery data were then com pared by One way ANOVA with Dunnett's Multiple Comparison Test to a theoretical recovery range of $80120 \%$ and $70130 \%$ for the Adda ELISA and the Serum ELISA, respectively. Data are pre sented as means \pm SEM. Significant differences are indicated as fol lows: $p<0.05\left({ }^{*}\right), p<0.01\left({ }^{* *}\right)$ and $p<0.001\left({ }^{* * *}\right)$. All statistical analyses were performed using GraphPad Prism 5.03 software. In case of results from single experiments, data is presented without any further calculations or statistical analysis.

\section{Results}

\subsection{Recovery of MC LR from spiked human serum in both ELISAs}

Fig. 2 summarizes the recovery data for MC LR using both ELISAs and the respective sample preparations (Fig. 1). A good recovery (approximately $80120 \%$ ) and reproducibility was achieved with MC LR concentrations ranging between 1 and $100 \mu \mathrm{g} / \mathrm{L}$ using the polyclonal Adda ELISA (Fig. 2A). These values were in accordance 
with the assay specifications of the manufacturer. With the mono clonal Serum ELISA, all samples spiked with MC LR (2 $200 \mu \mathrm{g} / \mathrm{L})$ were recovered, however all results overestimated the true concen tration by approximately $6080 \%$ (Fig. 2B). Additional testing with MC LR up to $1000 \mu \mathrm{g} / \mathrm{L}$ showed similar results for both ELISAs (data not shown).

\subsection{Recovery of $M C L R$ from spiked bovine serum and cell culture} medium using the Serum ELISA

Using the monoclonal Serum ELISA, specifically designed for human serum, the same recovery experiment described above was performed using fetal bovine serum (FBS) (Fig. 3A) and a typ ical cell culture medium (DMEM) supplemented with 10\% FBS (Fig. 3B). Again, the results overestimated the true concentrations. This effect was higher with higher FBS content of the sample matrix suggesting that undiluted bovine serum may produce increased matrix effects in the monoclonal Serum ELISA.

\subsection{Recovery of six MC congeners from spiked human serum in both} ELISAs

Six MC congeners (MC LR, YR, RR, LA, LW and LF) and an equimolar mixture of all six congeners were tested using both ELI SAs after application of the respective sample preparation method (Fig. 1). Here, three different concentration levels were chosen (1, 10 and $100 \mu \mathrm{g} / \mathrm{L}$ ). With both ELISAs differences were observed between the concentrations and the congeners (Fig. 4). In the poly clonal based Adda ELISA, MC LR, YR, RR as well as the mixture showed good recoveries, whereas for MC LA, LW and LF signifi
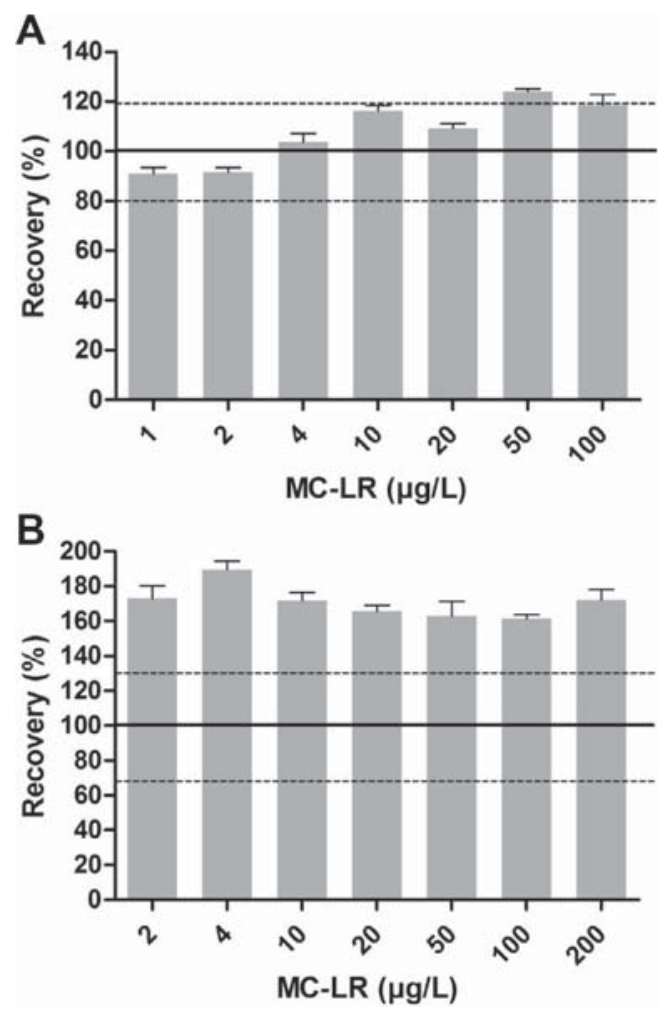

Fig. 2. Recovery of MC-LR from spiked human serum. MC-LR was spiked into human serum at different concentrations and samples were extracted according to the respective protocol for the polyclonal Adda-ELISA (A) and the monoclonal Serum-ELISA (B). Columns represent means from three independent samples $(n=3)+$ SEM analyzed in technical triplicates; dotted lines represent the expected recovery range (Adda-ELISA: $80-120 \%$, Serum-ELISA: $70-130 \%$ ).
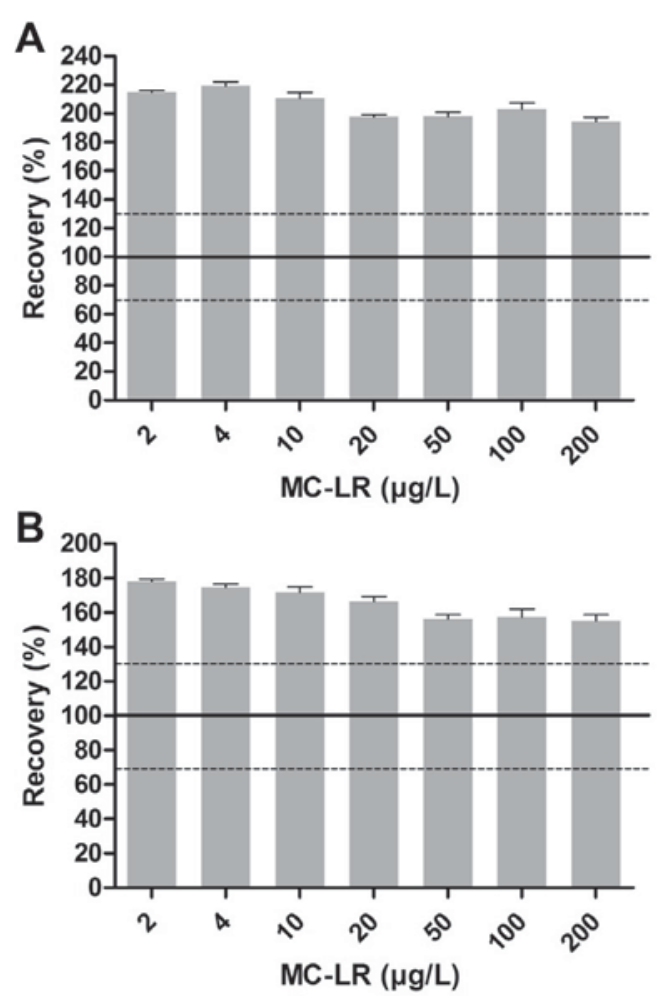

Fig. 3. Recovery of MC-LR from spiked FBS and cell culture medium using the serum-ELISA. MC-LR was spiked at different concentrations into bovine serum (FBS, A) and cell culture medium containing $10 \%$ FBS (B). Samples were extracted according to the protocol for the Serum-ELISA. Columns represent means from three independent samples $(n=3)$ analyzed in technical triplicates + SEM; dotted lines represent the expected recovery range of the monoclonal Serum-ELISA (70$130 \%)$

cant lower recoveries were observed (Fig. 4A). Furthermore, the recovery increased with increasing concentrations.

In the monoclonal Serum ELISA, all congeners as well as the mixture were reproducibly recovered, however for MC LR, YR, $R R$ and the mixture the true values were rather overestimated (Fig. 4B). Overall little differences in recovery were observable between the two concentrations of MC congeners tested (10 and $100 \mu \mathrm{g} / \mathrm{L})$.

\subsection{Inter laboratory comparison and storage vessel effects on recovery}

The initial comparison of the results obtained by the two labo ratories involved in this study demonstrated decisive differences in the recovery and precision of both ELISAs used. The latter differ ences were most prominent for MC LW and MC LF (data not shown). Therefore, a more profound inter laboratory comparison was performed using MC LR, LW and LF and the monoclonal based Serum ELISA in order to identify and eliminate the responsi ble parameter(s) for the differential outcome. The three main parameters that needed to be adapted were the methanol concen tration, type of sample container used, and the preparation of MC stocks (Table 1). Specifically the type of sample container used for the final dilutions prior to the ELISA had a major effect on the outcome and was analyzed with more detail. Fig. 5 shows the effect of different standard vessels on the recovery of MCs after short term storage for the selected three MC congeners with and without vortexing. For MC LR, a ranking could be established: glass $>$ HDPE $>$ LDPE $>$ PP (Fig. 5A). For PP the specific composition as represented here by the different suppliers appeared to be 

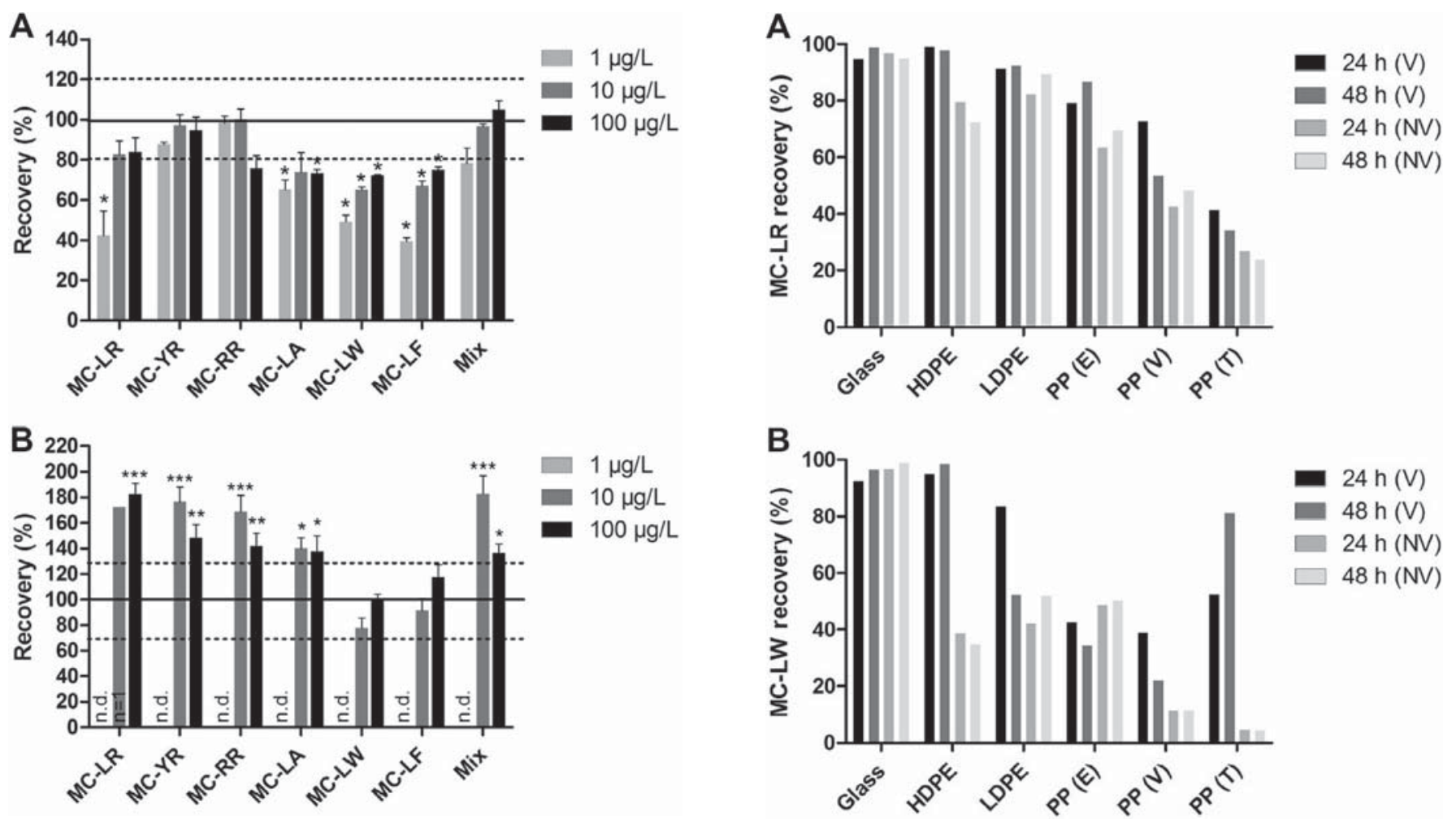

Fig. 4. Recovery of six MC congeners from spiked human serum. MC congeners were spiked into human serum in different concentrations: 1,10 and $100 \mu \mathrm{g} / \mathrm{L}$ Samples were extracted according to the respective protocol for the polyclonal Adda-ELISA (A) and the monoclonal Serum-ELISA (B). Columns represent means from three independent samples $(n=3)+$ SEM unless otherwise stated and were analyzed in technical triplicates; dotted lines represent the expected recovery range (Adda-ELISA: $80-120 \%$, Serum-ELISA: 70-130\%). One-way ANOVA with Dunnett's multiple comparison test with $p<0.05\left(^{*}\right), p<0.01\left(^{* *}\right)$ and $p<0.001\left(^{* * *}\right)$; note the $1 \mu \mathrm{g} / \mathrm{L}$ sample series were not determined (n. d.) in the Serum-ELISA (B).

crucial. The material ranking was found to be the same for MC LW and MC LF, however the overall recovery observed was MC LR $>$ MC LW > MC LF with all vessel types except those made of glass (Fig. 5B and C). Furthermore, mixing of the samples prior to pipetting resulted in higher recoveries of all three congeners from almost all vessel types. Longer contact of MC congeners to the stor age container generally resulted in higher loss of MC congeners from the solutions.

Subsequent to agreeing to a common procedure (Table 1 ) the ELISA analyses provided for similar results in both laboratories (Fig. 6)

\section{Discussion}

Toxic cyanobacterial blooms and the associated human expo sure require close monitoring in order to minimize the potential human health risks, especially in regions where water treatment for the preparation of drinking water is not as well established as it is in the more developed countries of Western Europe, North

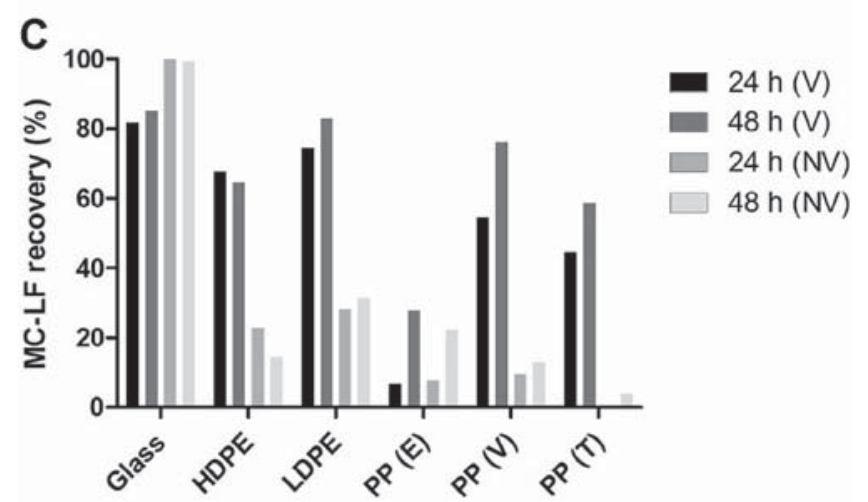

Fig. 5. Recovery of MCs (Monoclonal ELISA) after short-term storage in different standard vessels. MC-LR (A), MC-LW (B) and MC-LF (C) were spiked into ELISA diluent at $1 \mu \mathrm{g} / \mathrm{L}$ in glass vials and were then transferred into different vessels: PP (E) (polypropylene microcentrifuge tubes, very slightly opaque, Eppendorf), PP (T) (polypropylene microcentrifuge tubes, clear, Thomas Scientific), PP (V) (polypropylene microcentrifuge tubes, clear, VWR), low-density polyethylene (LDPE) bottles high-density polyethylene (HDPE) bottles, and glass vials with Teflon-lined caps. Monoclonal ELISA after $24 \mathrm{~h}$ and $48 \mathrm{~h}$ (stored at $4{ }^{\circ} \mathrm{C}$ between each analysis) after vortexing $(\mathrm{V})$ or non-vortexing $(\mathrm{NV})(n=1)$.

American, Australia and New Zealand. A prominent example is Lake Atitlan in Guatemala, where the first significant cyanobacte rial bloom occurred in 2008 and thereafter the blooms were increasingly serious with disastrous effects for the country $[33,34]$. In Uruguay, the Rio Uruguay and La Plata River estuarine

Table 1

Comparison of important parameters for both ELISAs.

\begin{tabular}{|c|c|c|}
\hline Parameter & Low recovery/precision & High recovery/precision \\
\hline Sample MeOH concentration & Up to $50 \%$ & $5 \%$ \\
\hline Type of sample container (for the preparation of MC dilutions prior to ELISA) & Polypropylene & Glass \\
\hline Preparation of MC stocks (molar absorption coefficient) ${ }^{a}$ & Different coefficients for the MC congeners & $39,800 \mathrm{~mol} \mathrm{~L}^{-1} \mathrm{~cm}^{-1}$ for all congeners \\
\hline
\end{tabular}

a Based on literature [27,28] and personal communication (Wayne Carmichael; Jussi Meriluoto; Linda Lawton). 


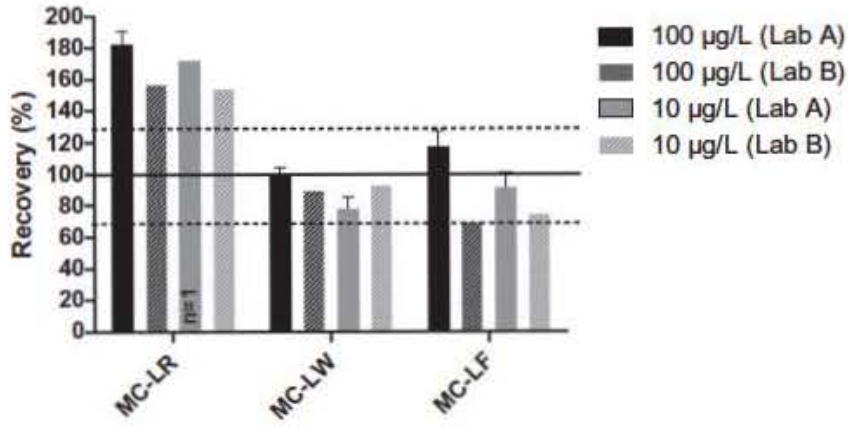

Fig. 6. Inter-laboratory comparison (Monoclonal Serum-ELISA). University of Konstanz (Lab A), Abraxis (Lab B); columns represent 6-12 independent samples analyzed in technical duplicates with SEM unless otherwise stated (Lab A) or 3 independent samples with SEM (Lab B).

system, which is the fifth largest in the world, is similarly affected $[33,35]$. For this system a program was initiated to exploit the potential of ELISAs to provide a low cost environmental analysis. This assay indeed enabled the first systematic monitoring of water from the Rio de la Plata at Montevideo and has been finally inte grated into a bi national effort to monitor the Rio de la Plata [36]. Similarly, an ELISA based monitoring system could be useful for the assessment of exposed inhabitants of toxic cyanobacterial bloom affected regions. Both parameters, MCs in the water and in the serum of exposed inhabitants would allow better assess ment of potential toxin uptake as well as provide for a basis to epi demiologically investigate the co occurrence of high incidences of diseases that could be associated with MC exposure.

Known quantitative detection methods for MCs include the pro tein phosphatase inhibition assay (PPIA), HPLC, LC MS and ELISA with the respective different levels of sensitivity and robustness [26.37 39]. The PPIA is very sensitive ( $L O D=0.010 .3 \mu \mathrm{g} / \mathrm{L}$ ) but prone to inhibitory matrix effects $[37,38,40]$. HPLC approaches are less sensitive with typical detection limits of $0.51 \mu \mathrm{g} / \mathrm{L}$ $[37,38]$. LC MS is very sensitive (LOD $0.020 .5 \mu \mathrm{g} / \mathrm{L}$ ) and highly specific, but requires expensive equipment and highly skilled per sonnel $[6,37,38]$. Both HPLC and LC MS largely depend on the availability of certified MC standards that then allow quantifying individual MC congeners. In contrast, ELISAs are generally highly sensitive $(L O D=0.020 .15 \mu \mathrm{g} / \mathrm{L})$, fairly robust to matrix effects $[26,37]$ and do not require certified standards of individual MC congeners. With respect to sensitivity and selectivity, a ranking of ELSA $>$ LC MS = HPLC was determined, whereas for costs, time and training required the ranking is reversed, i.e. LC MS > HPLC > ELSA [38]. Irrespective of selectivity and sensitivity, there is no single method available that is capable of detecting, lest quantifying, all potential MCs present in a given sample for reasons including lack of standards, limited sensitivity of detection for a given MC congener, and limitations in quantification due to loss during extraction or matrix effects etc. One of the potential solu tions in LC MS analyses, at least for the latter issue, is the use of stable isotope labeled MC congeners as internal standards and extraction tracers. Indeed, spiking analytical samples with a stable MC isotope prior to extraction allows to correct for sample loss during preparation and ion suppression during analysis [39]. How ever, in absence of commercially available stable isotope labeled MC congener, other intemal standards, such as thiol MC LR can be employed in HPLC and LC MS detection [16], albeit with the restrictions that thiol MC LR behaves differently during organic sample extraction than the parent MC LR.

Another important challenge that would allow much broader implementation of MC detection across the world is making MC analyses faster, cheaper, and more robust, making it feasible for regions without highly skilled personnel and sophisticated and expensive equipment. The ELISAs used in the study here represent such a method. The monoclonal based Serum ELISA is an espe cially fast and comfortable approach. Sample preparation is achieved within $30 \mathrm{~min}$ (compared to 23 days in the Adda ELISA), highly convenient (kit based) and needs only low cost equipment (microcentrifuge and plate reader).

In the study here, both chosen ELISAs in combination with their corresponding extraction procedures were able to detect MC LR in human serum, but with different recoveries. MC LR recovery was within the expected recovery range for all concentrations tested in the polyclonal Adda ELISA, whereas all concentrations were generally overestimated using the monoclonal Serum ELSA. A similar picture was obtained when using six different MC congen ers (i.e. MC LR, YR, RR, LA, LW and LF) and equimolar mixtures thereof. Of these congeners, MC LR, YR and RR were detected with reasonable recoveries in the polyclonal Adda ELISA while recoveries were overestimated in the monoclonal Serum ELISA, when compared to the expected recovery ranges. MC LA, LW and LF were underestimated using the polyclonal Adda ELISA, whereas they were detected correctly using the monodonal Serum ELISA, although the latter may represent a compensatory effect of general overestimation and lower recovery. Furthermore, the congeners were used as single toxins and as defined mixtures, resulting in a comparable detection of the congeners in the mixture compared to the single detection, indicating an equal or at least similar recognition of the chosen congeners by the antibody, which corroborates earlier data [30]. The observed differential recoveries of the different MCs are therefore not due to a differential recogni tion by the antibodies but rather due to a different behavior during the extraction procedure. This assumption is supported by the cur rent preliminary data on the effects of storage vessels and is con firmed by very recent data from our laboratory [41].

In summary, from the current data it can be concluded that both ELISAs are suitable for MC analysis in human serum. Although the correct quantification depends on the congeners present in a serum analyte, the ELISAs are at least suitable tools for a prelimin ary screening of samples. The recoveries obtained in the current study are in good accordance with data from other studies using similar techniques. For example an ELISA utilizing anti MC poly clonal serum showed similar cross reactivity of MC congeners (MC RR, 68\%; MC YR, 68\%, MC LW, 52\%, MC LF, 77\%) [33].

Additional testing was performed with the serum ELISA in order to determine its suitability for other applications. For this, the MC LR recovery experiment was repeated using bovine serum (FBS) instead of human serum. Here, the results showed high deviations from the real concentrations indicating high matrix effects from the serum. These results are in accordance with similar observa tions using dog serum (Rubio, unpublished data). From these results it can be concluded that the serum ELISA is specifically use ful for the analysis of human serum but that it may not be suitable for sera from other species as matrix effects were high.

Moreover, the suitability of the Serum ELISA for the detection of MCs in standard cell culture medium (DMEM containing $10 \%$ FBS) was investigated as this ELISA could serve to verify MC concentra tions employed in in vitro experiments. The MC concentrations commonly used in in vitro experimentations range from low nano molar to micromolar concentrations (e.g. up to $5 \mu \mathrm{M}[42]$ ) and thus represent concentrations that are within the detection range of the ELISA. However, based on the results obtained in the study here it must be concluded that the Serum ELSA is not suitable for this type of approach due to the high matrix related overestimation of the real MC concentrations.

In the human body, as well as in other exposed organisms including plants and prokaryotes and protists, MCs are at least in part covalently bound to proteins, specifically serine/threonine 
phosphatases and therefore, extracts from biological samples may reflect only freely available MCs [11,12,43 45]. This was also shown for human serum as the analysis of serum samples from MC exposed kidney dialysis patients revealed that both free and bound MC forms were present [14]. Moreover, it was demon strated that the standard methanol extraction procedure is not suitable for extracting covalently bound MCs [12,46]. In contrast, the data presented here reflect samples containing non covalently bound MCs only and thus do not represent the situation where both forms are present. Nevertheless, due to the type of interac tion, i.e. the covalent binding between the cystine residues of pro tein phosphatase 1 and 2 (PP) and the methyl dehydroalanine (Mdha) residue of the MCs [43 45], it can be assumed that the anti Adda antibodies used in both ELISAs should be able to detect free as well as bound MCs as long as the sample preparation method would provide for both types of molecules. Indeed, the lat ter assumption is corroborated by the fact that the anti Adda anti body can be used in Western blots for detecting MC covalently bound to protein phosphatases 1 and 2 [3].

Due to the detection range of the ELISAs, sample concentration and/or spiking may be required for detection of low levels of MCs in human serum. However, as the ELISA does not discriminate between MC congeners, yet MC congeners are subject to differential tissue/serum recoveries, only the addition of (a) stable isotope labeled internal MC standard(s) would allow more precise quantification, especially when coupled to subsequent LC MS analyses.

In conclusion, despite the research efforts of the past decades, further research is needed with respect to MC exposure, MC bio availability and subsequent toxicity in humans [2,39]. For this, direct monitoring of human exposure levels and associated effects is prerequisite. In the study presented here, it was possible to dem onstrate that ELISA is an affordable, robust and sensitive method that could serve to fulfill the toxin monitoring requirements men tioned above.

\section{Conflict of interest}

Dr. Dietrich reports grants from Marie Curie International Research Staff Exchange Fellowship within the 7th European Com munity Framework Program (PIRSES GA 2011 295223), during the conduct of the study; In addition, Dr. Dietrich has a patent "Conge ner independent detection of microcystin and nodularin congen ers". Patent \# PCT WO 01/18059 issued to Dr. Dietrich and The Regents, University of California System. Dr. Rubio is CEO of Abrax is LLC, the company who licensed Patent \# PCT WO 01/18059 and manufactures the two Adda ELISA employed in this study.

\section{Transparency Document}

The Transparency document associated with this article can be found in the online version.

\section{Acknowledgements}

This work was supported by a Marie Curie International Research Staff Exchange Fellowship within the 7th European Com munity Framework Program (PIRSES GA 2011 295223).

\section{References}

[1] A. Humpage, Toxin types, toxicokinetics and toxicodynamics, in: H.K. Hudnel (Ed.), Cyanobacterial Harmful Algal Blooms: State of the Science and Research Needs, Springer, New York, 2008, pp. 383-415.

[2] S. Merel, M.C. Villarín, K. Chung, S. Snyder, Spatial and thematic distribution of research on cyanotoxins, Toxicon 76 (2013) 118-131.
[3] S.J. Hoeger, D. Schmid, J.F. Blom, B. Ernst, D.R. Dietrich, Analytical and functional characterization of microcystins [Asp3]MC-RR and [Asp3, Dhb7]MC-RR: consequences for risk assessment?, Environ Sci. Technol. 41 (2007) 2609-26016.

[4] Y. Grosse, R. Baan, K. Kurt Straif, B. Secretan, F. El Ghissassi, V. Cogliano, Carcinogenicity of nitrate, nitrite, and cyanobacterial peptide toxins, Lancet Oncol. 7 (2006) 628-629.

[5] B.W. Ibelings, K.E. Havens, Cyanobacterial toxins: a qualitative meta-analysis of concentrations, dosage and effects in freshwater, estuarine and marine biota, Adv. Exp. Med. Biol. 619 (2008) 675-732.

[6] C. Robillot, J. Vinh, S. Puiseux-Dao, M.-C. Hennion, Hepatotoxin production kinetics of the cyanobacterium Microcystis aeruginosa PCC 7820, as determined by HPLC-mass spectrometry and protein phosphatase bioassay, Environ. Sci. Technol. 34 (2000) 3372-3378.

[7] D. Feurstein, K. Kleinteich, A.H. Heussner, K. Stemmer, D.R. Dietrich, Investigation of microcystin congener-dependent uptake into primary murine neurons, Environ. Health Perspect. 118 (2010) 1370-1375.

[8] D. Feurstein, K. Holst, A. Fischer, D.R. Dietrich, Oatp-associated uptake and toxicity of microcystins in primary murine whole brain cells, Toxicol. Appl. Pharmacol. 234 (2009) 247-255.

[9] A. Fischer, S.J. Hoeger, K. Stemmer, D.J. Feurstein, D. Knobeloch, A. Nussler, D.R. Dietrich, The role of organic anion transporting polypeptides (OATPS/SLCOs) in the toxicity of different microcystin congeners in vitro: a comparison of primary human hepatocytes and OATP-transfected HEK293 cells, Toxicol. Appl. Pharmacol. 245 (2010) 9-20.

[10] C. MacKintosh, J. Coggins, P. Cohen, Plant protein phosphatases. Subcellular distribution, detection of protein phosphatase $2 \mathrm{C}$ and identification of protein phosphatase $2 \mathrm{~A}$ as the major quinate dehydrogenase phosphatase, Biochem J. 273 (1991) 733-738.

[11] Y. Zilliges, J.C. Kehr, S. Meissner, K. Ishida, S. Mikkat, M. Hagemann, A. Kaplan, T. Boerner, E. Dittmann, The cyanobacterial hepatotoxin microcystin binds to proteins and increases the fitness of microcystis under oxidative stress conditions, PLoS ONE 6 (2011) e17615.

[12] S. Meissner, J. Fastner, E. Dittmann, Microcystin production revisited: conjugate formation makes a major contribution, Environ. Microbiol. 15 (2013) 1810-1820.

[13] E.M. Jochimsen, W.W. Carmichael, J.S. An, D.M. Cardo, S.T. Cookson, C.E. Holmes, M.B. Antunes, D.A. de Melo Filho, T.M. Lyra, V.S. Barreto, S.M. Azevedo, W.R. Jarvis, Liver failure and death after exposure to microcystins at a hemodialysis center in Brazil, N. Engl. J. Med. 338 (1998) 873-878.

[14] M. Yuan, W.W. Carmichael, E.D. Hilborn, Microcystin analysis in human sera and liver from human fatalities in Caruaru, Brazil, 1996, Toxicon 48 (2006) 627-640.

15] S. Pouria, A de Andrade, J. Barbosa, R.L. Cavalcanti, VT.S. Barreto, C.J. Ward, W. Preiser, G.K. Poon, G.H. Neild, G.A. Codd, Fatal microcystin intoxication in haemodialysis in Caruaru, Brazil, The Lancet 352 (1998) 21-26.

[16] J.L. Smith, G.L. Boyer, Standardization of microcystin extraction from fish tissues: a novel internal standard as a surrogate for polar and non-polar variants, Toxicon 53 (2009) 238-245.

[17] R.M. Soares, M. Yuab, J.C. Servaites, A. Delgado, V.F. Magalhaes, E.D. Hilborn, W.W. Carmichael, S.M.F.O. Azevedo, Sublethal exposure from microcystins to renal insufficiency patients in Rio de Janeiro, Brazil, Environ. Toxicol. 21 (2006) 95-103.

[18] W.W. Carmichael, S.M.F.O. Azevedo, JS. An, R.R. Molica, E.M. Jochimsen, S. Lau, K.L. Rinehart, G.R. Shaw, G.K. Eaglesham, Human fatalities from cyanobacteria: chemical and biological evidence for cyanotoxins, Environ. Health Perspect. 109 (2001) 663-668.

[19] S.M.F.O. Azevedo, W.W. Carmichael, E.M. Jochimsen, K.L. Rinehart, S. Lau, G.R. Shaw, G.K. Eaglesham, Human intoxication by microcystins during renal dialysis treatment in Caruaru-Brazil, Toxicology 181-182 (2002) 441-446.

[20] E.D. Hilborn, R.M. Soares, J.C. Servaites, A.G. Delgado, V.F. Magalhães, W.W Carmichael, S.M. Azevedo, Sublethal microcystin exposure and biochemical outcomes among hemodialysis patients, PLoS ONE 8 (2013) e69518.

[21] D.R. Dietrich, A. Fischer, C. Michel, S.J. Hoeger, Toxin mixture in cyanobacterial blooms-a critical comparison of reality with current procedures employed in human health risk assessment, Adv. Exp. Med. Biol. 619 (2008) 885-912.

[22] A.H. Heussner, L. Mazija, J. Fastner, D.R. Dietrich, Toxin content and cytotoxicity of algal dietary supplements, Toxicol. Appl. Pharmacol. 265 (2012) 263-271.

[23] M.L. Saker, A.-D. Jungblut, B.A. Neilan, D.F.K. Rawn, V.M. Vasconcelos, Detection of microcystin synthetase genes in health food supplements containing the freshwater cyanobacterium Aphanizomenon flos-aquae, Toxicon 46 (2005) 555-562.

[24] J. Chen, P. Xie, L. Li, J. Xu, First identification of the hepatotoxic microcystins in the serum of a chronically exposed human population together with indication of hepatocellular damage, Toxicol. Sci. 108 (2009) 81-89.

25] Y. Li, J.A. Chen, Q. Zhao, C. Pu, Z. Oiu, R. Zhang, W. Shu, A cross-sectiona investigation of chronic exposure to microcystin in relationship to childhood liver damage in the Three Gorges Reservoir Region, China, Environ. Health Perspect. 119 (2011) 1483-1488.

26] J.S. Metcalf, G.A. Codd, Analysis of cyanobacterial toxins by immunological methods, Chem. Res. Toxicol. 16 (2003) 103-112.

[27] K. Harada, K. Matsuura, M. Suzuki, M.F. Watanabe, S. Oishi, A.M. Dahlem, V.R. Beasley, W.W. Carmichael, Isolation and characterization of the minor components associated with microcystins LR and RR in the cyanobacterium (blue-green algae), Toxicon 28 (1990) 55-64. 
[28] J. Meriluoto, L. Spoof, Preparation of standard solutions of microcystin-LR for HPLC calibration, in: J. Meriluoto, G.A. Codd (Eds.), Toxic: Cyanobacterial Monitoring and Cyanotoxin Analysis, Acta Academiae Aboensis. Ser. B, Mathematica et physica, Åbo Akademi University Press, 2005, pp. 65-68.

[29] R.W. Burke, R. Mavrodineanu, Acidic potassium dichromate solutions as ultraviolet absorbance standards, J. Res. Natl. Bur. Stand. A Phys. Chem. 80A (1976) 631-636.

[30] W.J. Fischer, I. Garthwaite, C.O. Miles, K.M. Ross, J.B. Aggen, A.R. Chamberlin, N.R. Towers, D.R. Dietrich, Congener-independent immunoassay for microcystins and nodularins, Environ. Sci. Technol. 35 (2001) 4849-4856.

[31] EPA, Technology Brief: Immunoassay Test Kits for Microcystins (EPA/600/S/12/ 511), 2012.

[32] M.G. Weller, A. Zeck, A. Eikenberg, S. Nagata, Y. Ueno, R. Niessner, Development of a direct competitive microcystin immunoassay of broad specificity, Anal. Sci. 17 (2001) 1445-1448.

[33] M. Pírez G. Gonzalez-Sapienza, D. Sienra, G. Ferrari, M. Last, J.A. Last, B.M. Brena, Limited analytical capacity for cyanotoxins in developing countries may hide serious environmental health problems: simple and affordable methods may be the answer, J. Environ. Manage. 114 (2013) 63-71.

[34] T.S. Harvey, Cyanobacteria blooms: Maya peoples between the politics of risk and the threat of disaster, Med. Anthropol. 31 (2012) 477-496.

[35] L. De Leon, J.S. Yunes, First report of a microcystin-containing bloom of the cyanobacterium microcystis aeruginosa in the La Plata River, South America, Environ. Toxicol. 16 (2001) 110-112.

[36] B.M. Brena, L. Díaz, D. Sienra, G. Ferrari, N. Ferraz, U. Hellman, G. GonzalezSapienza, J.A. Last, ITREOH building of regional capacity to monitor recreational water: development of a non-commercial microcystin ELISA and its impact on public health policy, Int. J. Occup. Environ. Health 12 (2006) 377385.

[37] T.A. Msagati, B.A. Siame, D.D. Shushu, Evaluation of methods for the isolation, detection and quantification of cyanobacterial hepatotoxins, Aquat. Toxicol. 78 (2006) 382-397.
[38] P.R. Hawkins, S. Novic, P. Cox, B.A. Neilan, B.P. Burns, G. Shaw, W. Wickramasinghe, Y. Peerapornpisal, W. Ruangyuttikarn, T. Itayama, T. Saitou, M. Mizuochi, Y. Inamori, A review of analytical methods for assessing the public health risk from microcystin in the aquatic environment, J. Water SRT Aqua 54 (2005).

[39] S. Merel, D. Walker, R. Chicana, S. Snyder, E. Baurès, O. Thomas, State of knowledge and concerns on cyanobacterial blooms and cyanotoxins, Environ. Int. 59 (2013) 303-327.

[40] V.P.S. Almeida, K. Cogo, S.M. Tsai, D.H. Moon, Colorimetric test for the monitoring of microcystins in cyanobacterial culture and environmental samples from southeast Brazil, Braz. J. Microbiol. 37 (2006) 192-198.

[41] A.H. Heussner, S. Altaner, L. Kamp, F. Rubio, D.R. Dietrich, Pitfalls in microcystin extraction and recovery from human blood serum, Chem-Biol. Interact. 223 (2014) 87-94.

[42] A. Fischer, S.J. Hoeger, K. Stemmer, D.J. Feurstein, D. Knobeloch, D. Nussler, D.R. Dietrich, The role of organic anion transporting polypeptides (OATPs/SLCOs) in the toxicity of different microcystin congeners in vitro: a comparison of primary human hepatocytes and OATP-transfected HEK293 cells, Toxicol. Appl. Pharmacol. 245 (2010).

[43] R.W. MacKintosh, K.N. Dalby, D.G. Campbell, P.T.W. Cohen, P. Cohen, C. MacKintosh, The cyanobacterial toxin microcystin binds covalently to cysteine-273 on protein phosphatase 1, FEBS Lett. 371 (1995) 236-240.

[44] M. Runnegar, N. Berndt, S.M. Kong, E.Y.C. Lee, L.F. Zhang, In vivo and in vitro binding of microcystin to protein phosphatase 1 and $2 \mathrm{~A}$, Biochem. Biophys. Res. Commun. 216 (1995) 162-169.

[45] M. Craig, H.A. Luu, T.L. McCready, D. Williams, R.J. Andersen, C.F. Holmes, Molecular mechanisms underlying he interaction of motuporin and microcystins with type- 1 and type-2A protein phosphatases, Biochem. Cell Biol. 74 (1996) 569-578.

[46] B. Ernst, L. Dietz, S.J. Hoeger, D.R. Dietrich, Recovery of MC-LR in fish liver tissue, Environ. Toxicol. 20 (2005) 449-458. 